\title{
ReviewArticle,
}

\section{Healthcare Providers' Perceptions on Desired Attributes for Mhealth Apps to Improve Adolescents and Young Adults Living with HIV Healthcare Outcomes in Pandemics like COVID-19: A Qualitative Evidence Review}

\author{
Annah Rufu ${ }^{1,2,} K_{\text {Chitindingu }}{ }^{1}$, S Chinofunga ${ }^{1}$, O Mugurungi ${ }^{2}$, B Dupwa $^{2}$ \\ ${ }^{1}$ Chinhoyi University of Technology \\ ${ }^{2}$ Ministry of Health and Child Care, Zimbabwe \\ Email Address: annah95@gmail.com
}

\begin{abstract}
:
Aim:

There was concern that the COVID-19 pandemic would adversely affect adolescents and young adults' HIV and TB program services in Zimbabwe. This review explores perceptions of healthcare providers (HcPs) on desirable attributes for mobile-health applications to improve adolescents and young adults living with HIV healthcare outcomes in emergencies and pandemics like COVID 19.
\end{abstract}

Background:

Studies in all study settings have reported encouraging potential for Mobile-Health (mHealth) technologies in improving health outcomes by preventing treatment interruption. However, literature bears little evidence of use in information dissemination in emergencies and pandemics like COVID 19. It was rather more on monitoring and evaluation. Paying little attention to information and mental health support in emergencies and pandemics as one of the key drivers of innovation adoption has been widely cited as a notable mHealth limitation.

Methods:

This systematic review navigated through English authored peer-reviewed publications from health, computer engineering and social science repositories as guided by the Preferred Reporting Items for Systematic Reviews and Meta-Analyses (PRISMA) statement. The search was conducted between February 2018 and April 2021 thus, yielding eleven (11) complete-text publications.

\section{Results:}

HcPs in the reviewed literature have used mobile-technology applications for monitoring and management of sexual $(18.2 \%)$, reproductive \& maternal health (9.1\%), HIV (45.5\%), Malaria $(9.1 \%)$ and other chronic diseases $(36.4 \%)$. Commonly mentioned sub-features in $72.7 \%$ of the studies identified motivation and trust attributes as desirable, $36.4 \%$ highlighting synchronism of technology, adaptability of App with different mobile-phones recorded in $45.5 \%$ of the studies while $63.6 \%$ value the fit-for-purpose attribute. These features were cited as desirable with little evidence of the geo-social influence of the users nor socioeconomic setting.

\section{Conclusion:}

The study exposed the HcPs' perceptions to the mobile-technology attributes as customised requirements, process requirements, moderating information-technology constructs and intervening \& dependent requirements. Geospatial setting and income status of the country are less important in shaping the perceptions of HcPs as users of the mobile-technology.

Keywords: Healthcare provider, mHealth, COVID-19, HIV, pandemic, App attributes/features. 

and Young Adults Living with HIV Healthcare Outcomes in Pandemics like COVID-19: A Qualitative Evidence Review

\section{Introduction:}

The World Health Organization (WHO) on March $11^{\text {th }} 2020$, declared a global pandemic of coronavirus disease 2019 (COVID-19), caused by a novel coronavirus named severe acute respiratory syndrome coronavirus 2 (SARS-CoV-2). On the $31^{\text {st }}$ of March 2020 Zimbabwe announced its first 21days of COVID-19 lockdown. Measures effected by the government of Zimbabwe and other countries globally were not only limited to quarantine and restricted movement by general population. All of this led to concerns that countries with high burdens of tuberculosis (TB) and human immunodeficiency virus/acquired immune deficiency syndrome (HIV/AIDS) like Zimbabwe might be unable to provide uninterrupted and quality healthcare services to their patients especially among adolescents and young adults living with HIV (AaYALHIV).

There was concern that the COVID-19 pandemic would adversely affect the gains of AaYALHIV TB and HIV program services in Zimbabwe. The AaYALHIV is one of the special group under monitoring due to its poor management outcomes like poor viral load suppression, poor adherence, poor retention in care. The COVID-19 lockdown was a threat to the gains on HIV nationwide especially AaYALHIV. Hence this review which explores perceptions of healthcare providers (HcPs) on desirable attributes for mobile-health applications to improve adolescents and young adults living with HIV healthcare outcomes in emergencies and pandemics like COVID-19.

Vital Wave Consulting (2009) acknowledge the increasing interest especially in the developing world in using mobile technology to improve health outcomes. The dynamism in the mHealth field has seen an upsurge in the variety and design of applications (apps). Notable applications in resource-constrained setting include health education and information, data gathering, diagnostic and treatment services, off-site monitoring, epidemiological surveillance as well as healthcare provider communication and training.

The International Telecommunications Union (2013) posits that evidence-based interventions have increasingly been enhanced by access to mobile technology. Omachonu and Einspruch (2010) estimate there will be about 6.1 billion global mobile technology users by 2020 . According to the United Nations (2010), mHealth uses the functions of a mobile device, but most commonly relies on the download of mobile apps to help support health care delivery. The design of these Apps varies in functionality making room for suitability with a range of healthcare services including monitoring, skills practice, information, education and communication.

The World Health Organisation (2011) merits mHealth in as far as it enhances efficiency, lower communication costs, anonymity, availability, increasing health service capability, customised content and immediate support. Mobile technology use has greatly reduced geospatial barriers that have for a long time hindered healthcare support to difficulty-to-reach groups.

Venkatesh, Thong and Xu (2012) in their content analysis of Apps on the market noted with concern the disparities between evidence-based clinical guidelines and the architecture of downloadable Apps. Tomlinson et al (2013) weighed in to the same subject by observing that merely four in the 27 Apps corroborated effectiveness with evidence of patient-based outcomes. The bulk of the Apps hardly acknowledge information sources to allow user to verify authenticity.

Healthcare solutions can only be useful if they are of high quality, uninterrupted, cost effective, safe, efficient, and of positive impact to health outcomes. Venkatesh, Speier and Morris (2002) and Svoronos et al (2010) argued that in designing the healthcare stakeholders' mobile technology needs and requirements, it is advisable to cater for a range of applications from treatment to outreach. Thus, this review explores perceptions of healthcare providers on desirable attributes for mobile health Apps. The focus of this review is on mobile phone apps only (as opposed to broad mHealth and eHealth interventions) and will include a wide remit of publication types.

\section{Methods:}

\subsection{Search Strategy}

The systematic review was a three-step process starting with assessing abstracts and titles according to the inclusion criteria followed by assessing full text according to the inclusion criteria before potential sampling from the included studies. Guided by this search map, conduction and navigation was done through English authored peer-reviewed publications from health, computer engineering and social science repositories as guided by the Preferred Reporting Items for Systematic Reviews and Meta-Analyses (PRISMA) statement. Searching of the Pubmed/Medline, CAB Global Health, Web of Science, and INSPEC databases was done. The 
search reviewed complete-text publications from January 2014 to October 2018. Next, the search scanned credible institutional databases that included WHO publication database, Health UnBound (HUB) Content Library, and Royal Tropical Institute resource database. The literature recorded feedback from healthcare providers (as some of the end-users of the technology) on their perceived desirable attributes of mobile phone applications for improved clinical outcomes including HIV. As mHealth is a rapidly developing topic, it is likely that many studies are presented at conferences but not yet as journal articles. Searching was done for ISI Index of Conference Proceedings and Embase for such conference papers that might not be found elsewhere.

\subsection{Inclusion and Exclusion Criteria}

For this review, the phenomenon of interest is healthcare workers' perceptions and experiences of their use of mHealth technologies to provide and support primary healthcare services. Healthcare providers will include everyone involved in providing primary healthcare services, including professionals, paraprofessionals, lay health workers, and others, such as managerial and supervisory staff. The study did not assess the use of mHealth for patients' self-management of health and illnesses, as the healthcare provider themselves will not be using this technology and therefore their perceptions will not be of direct relevance to this study.

MHealth technologies thus refer to mobile devices that are used to create, store, retrieve and transmit data in real time between users. To qualify as mHealth, the publications either had to explicitly mention the term 'mHealth', or specify both the term 'health' and any one of the following search terms: handheld computer, mobile phone, cellular phone, mobile device, patient monitoring device, mobile telemedicine, MP3 player, mobile operating system technology, 3G, SMS, text message, IVR, interactive voice response, GPS or global positioning system.

From this broad search strategy, 203 articles were retrieved. Repeat citations across sources were picked and eliminated while inclusion the criteria accommodated bibliographies and some credible institutional literature. Subjection of this full-text article residue was done to intense review with specific focus on those mentioning healthcare providers' perceptions of desirable features for mobile technology for use in healthcare services while discarding those that did not meet the search criteria. Exclusions ranged from systematic reviews, policy briefs, commentaries and any other summary-type literature. Exclusion of articles lacking full-text helped further narrow the focus to articles reporting healthcare providers' perceptions on their expectations of attributes in a user-friendly mobile health App. A final list of $\mathbf{1 1}$ articles (Figure 1) yielded for perception analysis.

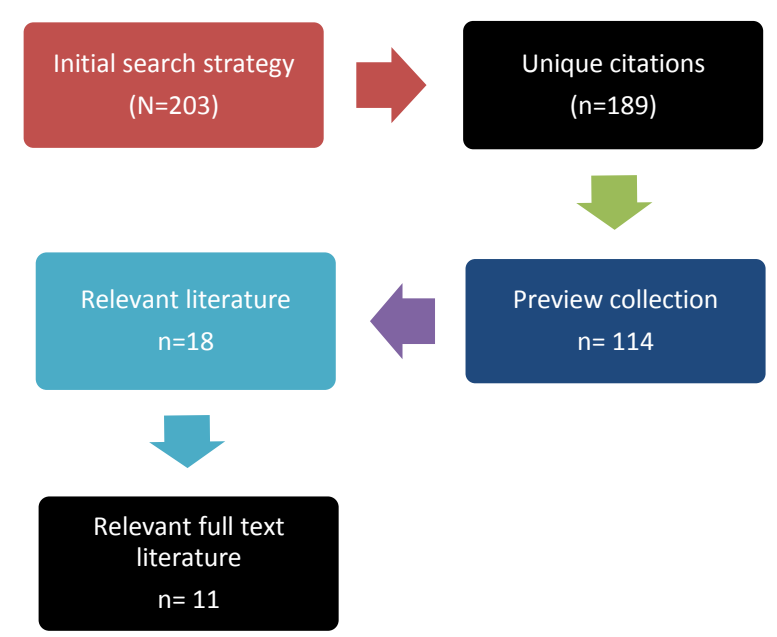

Figure1: Summary of search strategy

\subsection{Data Collection}

Braun et al (2013)'s strategy of data collection was adopted to systematically code articles. The characterisation of the literature is packaged to describe the following topics: study design and scope, methods, sampling, target group/participants, findings, perceived desired mHealth attributes, type of mobile platform, location, health issues addressed, information architecture and conclusions.

\subsection{Data extraction \& Analysis}

The study adopted the Best-fit framework synthesis developed by Carroll and colleagues (Carroll et al, 2013). The process will comprise the following:

Step 1: A theoretical model as framework to assess the perceptions and experiences of those who use mHealth:

The Best-Fit theoretical model that was considered to capture the salient aspects of the perceptions and experiences of healthcare providers and others, on their use of mHealth technology to deliver primary healthcare services was identified. 


\section{Step 2: Development of the data extraction form:}

The first part of this form comprised drafting and extracting the study characteristics (compressed into four themes as (i) individual requirements, (ii) process requirements, (iii) moderating IT constructs and, (iv) intervening and dependent constructs).

\section{Step 3: Coding of the full texts:}

Manually coding in Word was done, the full texts using the themes developed in Step 2. Though it is commonly found that the author findings presented in the Results and Discussion sections contain the data to be coded, all sections were read for possible coding.

\section{Step 4: Appraisal of study quality}

The core quality criterion that a study must meet to be eligible for inclusion, is that it must have used qualitative data collection - and analysis methods. An appraisal form, was developed based on (i) the Critical Appraisal Skills Programme (CASP) (Atkins 2008), and (ii) the methods proposed by Carroll and her colleagues (Carroll 2012) to assess the quality of the included studies.

Table 1 offers a summary of the study scope.

Table 1: Summary of study scope (Adapted from Braun et al, 2013)

\begin{tabular}{|l|l|l|l|}
\hline Category & Sub-category & $\begin{array}{l}\text { Results } \\
(\mathbf{n})\end{array}$ & $\begin{array}{l}\text { Results } \\
(\%)\end{array}$ \\
\hline Region & S.S. Africa & 6 & 54.5 \\
\hline & Asia & 1 & 9.1 \\
\hline & America & 4 & 36.4 \\
\hline \multirow{2}{*}{$\begin{array}{l}\text { Health Issue } \\
\text { addressed }\end{array}$} & $\begin{array}{l}\text { Sexual, Reproductive, } \\
\text { Maternal \& Child Health }\end{array}$ & 1 & 9.1 \\
\cline { 2 - 4 } & HIV/AIDS & 5 & 45.5 \\
\cline { 2 - 4 } & Other chronic diseases & 4 & 36.4 \\
\cline { 2 - 4 } & Malaria & 1 & 9.1 \\
\hline \multirow{2}{*}{$\begin{array}{l}\text { Type of } \\
\text { Platform }\end{array}$} & Android/iOS & 10 & 66.7 \\
\hline & & & \\
\hline & Windows & 3 & 20.0 \\
\hline & $\begin{array}{l}\text { Combination } \\
\text { (Android/iOS -Windows }\end{array}$ & 2 & 13.3 \\
\cline { 2 - 4 } & & & \\
\hline
\end{tabular}

\section{Results and discussion:}

\subsection{Scope of Research}

The study reviewed 11 articles (Table 1) of the 18 relevant literature as some articles narrated patient perceptions and not healthcare provider views as delineated for this study. The majority of the reviewed articles recorded perceptions from SubSaharan Africa $(n=6 ; 54.5 \%), 9.1 \%(n=1)$ from Asia while views from America attributed for $36.4 \%(n=4)$ of the review. HIV/AIDS $(45.5 \%)$ and other chronic diseases $(n=4 ; 36.4 \%)$ dominated the issues that were addressed by the reviewed studies. A few cases of malaria and tuberculosis $(\mathrm{n}=1$ each; $9.1 \%)$ were cited in some studies. Most of the mobile technology in reviewed studies used Android/iOS $(\mathrm{n}=10 ; 66.7 \%)$ as operating system, $20 \%(n=3)$ of the mobile phones were powered by Windows while $13.3 \%$ of the studies reported a combination of operating systems.

\subsection{Research Designs and Methods}

Results summarised in Table 2 show that $45.5 \%$ $(n=5)$ of the reviewed studies employed qualitative methodology while equal numbers $(n=3 ; 27.3 \%)$ used either quantitative or mixed methods. Experimental designs dominated $(45.5 \% ; \mathrm{n}=5)$ followed by quasi-experimental $(n=4 ; 36.4 \%)$ with non-experimental $(\mathrm{n}=2 ; 18.2 \%)$ being the least popular design among the reviewed literature. The reviewed studies used a variety of data collection tools to gather perceptions of healthcare providers' desired attributes in a mobile App for health interventions with some employing multiple tools. About a third $(n=5 ; 33.3 \%)$ of the reviewed literature reported to have used interviews (Key Informant \& Semi-structured) followed by off-line surveys $(26.7 \%)$. FGDs, on-line surveys and questionnaires were equally used $(n=2 ; 13.3 \%$ each) in the reviewed studies. Thompson et al (2018) reported an exploratory qualitative study of Health Surveillance Assistants in Malawi who piloted use of a mobile phone-based application called SL eCCM. This mobile App was designed for community case management. Prinja et al (2018) presented survey results of an economic analysis they carried out in India for a ReMiND project that used a mobile application for maternal health monitoring. Engle et al (2015)'s facility cluster randomised controlled design trial sampled perceptions from 40 Ghana Health Services Centres, healthcare providers and facility managers. This was a HIV care and treatment project aimed at improving ART adherence, viral load suppression, retention in care and condom use. This study by Engle and colleagues is one of the few inquiries giving more in-depth insights on 
cost effectiveness as one of the desirable attributes. Generally, the studies showed a common tendency toward increased use of experimental research designs. Differences in article reporting standards is attributed to variations in style requirements across the fields of medicine, computer studies and social science.

Table 2: Design \& methodological construct of reviewed literature

\begin{tabular}{|c|c|c|c|}
\hline Category & Sub-category & $\begin{array}{l}\text { Results } \\
\text { (n) }\end{array}$ & $\begin{array}{l}\text { Results } \\
(\%)\end{array}$ \\
\hline \multirow{3}{*}{$\begin{array}{l}\text { Research } \\
\text { Methods }\end{array}$} & Qualitative & 5 & 45.5 \\
\hline & Quantitative & 3 & 27.3 \\
\hline & Mixed & 3 & 27.3 \\
\hline \multirow{3}{*}{$\begin{array}{l}\text { Research } \\
\text { Designs }\end{array}$} & Non-experimental & 2 & 18.2 \\
\hline & Quasi-experimental & 4 & 36.4 \\
\hline & Experimental & 5 & 45.5 \\
\hline \multirow[t]{5}{*}{ Tools } & $\begin{array}{l}\text { Focus Group } \\
\text { Discussions (FGDs) }\end{array}$ & 2 & 13.3 \\
\hline & On-line survey & 2 & 13.3 \\
\hline & Off-line survey & 4 & 26.7 \\
\hline & Interviews (KII \& SSI) & 5 & 33.3 \\
\hline & Questionnaires & 2 & 13.3 \\
\hline
\end{tabular}

\subsection{Healthcare Provider perceptions of desired mobile App attributes}

The need to align people, processes, and technology to develop useful mHealth solutions is the guiding principle in system implementation that forms the three pillars (individual requirement, process requirement and intervening variables) of the Best-Fit framework (Carroll et al, 2013) preferred for this review. According to Goodhue and Thompson (1995), fit refers to the capability of technology to meet demands of a task measured in terms of task characteristics and technology characteristics. According to Overby (2008), moderating IT constructs are posited to positively moderate the potentially negative influence of individual and process requirements to achieve fit. Process requirements in our case refer to characteristics of a physical healthcare process that have to be fulfilled by virtualization, i.e., by a mHealth application. Individual requirements describe factors that influence the acceptance of the mHealth application. Discussion on individual attitudes regarding the physical process was not done since the assumption was that the necessity of the physical process is given.

\subsubsection{Individual requirements}

Behavioural control: Mburu, Franz \& Springer (2013) describe Behavioural control to an individual's perception on ability to perform a task of interest (Can I do?). From the reviewed literature, $54.5 \%$ cited that usability and userfriendliness are very important for a positive perception on this ability. Biello and Mayer (2018), Saberi et al (2016), Blanas et al (2014) and Levy et al (2015) all concur that an artefact should be usable and user-friendly to meet this requirement.

Subjective norms: Subjective norms refer to perceived social pressure to perform or not to perform a task (Must I Do?). Though there is social pressure to adopt innovative IT solutions, most healthcare professionals hesitate to adopt such technologies due to fear of violating code of ethics (Mburu, Franz \& Springer, 2013). Another perspective on "Must I do?" concerns benefits of a virtual process in comparison to a physical process, or, in other words, the necessity to use the virtual process such as mHealth. However, regarding the healthcare structure in most developing countries, mHealth can help to overcome problems of physical processes, e.g., improve access to healthcare at reduced cost and travel time. About $45.5 \%(n=5)$ of the reviewed literature placed emphasis on subjective norms using different expressions. In a survey by Call et al (2015), about $23 \%$ of the mobile phone users mentioned "convenience" as an important attribute. The Malawi Health Surveillance Assistants (HSAs) in Thompson et al (2018)'s exploratory study expressed the desire for the mobile App to enable them "retrieval of patient records" as an expression of convenience.

Attitude: According to Mburu, Franz \& Springer (2013), attitude refers to the degree to which a person has a positive or negative judgment of certain behaviour (Willing to do?). Two factors that may determine an individual's attitude are motivation and trust. The review found that $72.7 \%$ of the studies considered highly the motivational and trust attributes of a mobile App. Saberi et al (2016), Levy et al (2015) cited that if using the (virtualized) mHealth process promises to be, e.g., faster, simpler, and more cost effective, individuals are motivated to use it. Another important factor is trust. Trustworthiness of an application includes security, privacy, and safety; the application has to 
work as expected even in case of unwanted events such as failures or attacks (Mburu, Franz \& Springer, 2013). Individuals will only use a mHealth application if it is trustworthy.

\subsubsection{Process requirements:}

Sensory requirements: This refers to senses of touch, smell, sight, taste and hearing. These senses are important in healthcare processes particularly those involving diagnosis and treatment. About $27.3 \% \quad(n=3)$ of the reviewed literature acknowledged the desirability of the mobile App to offer voice calls, text messages and visuals in line with sensory requirements. Levy et al (2015) and Saberi et al (2016), in their studies noted that users appreciated to "receive App related alerts and messages". In fact, Okuboyejo and Eyeson (2014) did a qualitative evaluation in a Nigerian project in which the efficacy of sms alerts complimented by voice calls. Overby (2008) argues that if a process requires use of senses such as smell, taste or touch, then it would benefit more from physical context, which make them difficult to virtualize.

Relationship: Relationship is the need for participants of healthcare scenario such as a doctor and a patient to interact with one another. While in a face-to-face interaction cues such as gestures, posture, and inflection are part of communication, in virtual interaction such cues may be lacking. In $27.3 \% \quad(n=3)$ of the reviewed literature, the interactive options of the mobile App were expressed by healthcare providers. Though some degree of interaction can be achieved through use of multimedia technologies such as videoconferencing, available devices and infrastructure may be a limiting factor. In Malawi, Thompson and colleagues (2018) gathered from HSAs that the desired the mobile App to facilitate networking with their peers and supervisors. This was a HIV care and treatment project aimed at improving ART adherence, viral load suppression, retention in care and condom use. Similar expectations were expressed in Saberi et al (2016)'s focus group discussions in USA. Levy et al (2015)'s study weighed in with another dimension of the desirability for social networking of users via that mobile health App.

Identification and data protection: Mburu, Franz \& Springer (2013) posit that in a physical process the participants involved can easily identify each other but in a virtual environment, it is difficult to certainly confirm the identity of the remote entity. Another aspect is privacy or data protection
(Vashist, Schneider and Luong, 2014). In the physical world, we know which information we disclose to whom and in which situation. If we do not meet physically, it becomes harder to control who should know what about us. Both identification and privacy are important factors regarding trust in a virtual process as expressed in $27.3 \%$ of the reviewed literature.

Synchronism: Physical processes tend to be highly synchronous because two parties communicate with each other with minimal or no delay. However, in a virtual environment some tasks that require real-time processing such as tele surgery and sensor-based patient monitoring may not be easy to achieve. About $45.5 \%$ of the studies review that users aspire to have mobile Apps that are fast-paced (Levy et al, 2015), adaptability to various mobile phone devices (Vashist, Schneider and Luong, 2014 and; Levy et al, 2015). Saberi et al (2016) go on to cite "little overlaps with other Apps" as an element of ensuring speedy functionality.

\subsubsection{Moderating information technology constructs:}

Representation: Representation refers to the capability to use IT to simulate sensory experience and enable interaction between remote participants. The former can be achieved by use of sensors, while the latter by means of multimedia technology such as audio or video. Further, the representation of information as well as functionality strongly influences usability and user experience. About $36.4 \%$ of the studies mentioned the desire for multi-media functions on the mobile App. Levy et al (2015) called it "diverse interactive functions", Saberi et al (2016) mentioned "unlimited access to entertainment and informative" while Biello and Mayer (2018) expressed "App content and functionalities" among key attributes.

Reach: Using mHealth as a virtualized process clearly provides benefits regarding challenges of accessing healthcare (Mburu, Franz \& Springer, 2013). A useful mHealth solution can potentially reduce the need for physical interaction between doctors and patients (Saberi et al, 2016). Almost a third $(27.3 \%)$ of the studies expressed that availability and connectivity are essentials for a reliable mobile App. In addition, IT can support reach by ensuring availability (Blanas et al, 2014) of information independent of time. The challenge is how to ensure reach using the available devices 
Annah Rufu et al. / Healthcare Providers' Perceptions on Desired Attributes for Mhealth Apps to Improve Adolescents and Young Adults Living with HIV Healthcare Outcomes in Pandemics like COVID-19: A Qualitative Evidence Review

and infrastructure.

Security and Privacy Mechanisms: Security and privacy features have to be integrated into a mHealth solution to ensure that the corresponding process requirements are met. Fulfilling these requirements is essential for the trustworthiness of the application. Another example is the enforcement of integrity of information; doctors will not use a mHealth application if they cannot be sure to get the right information regarding a patient. Thus, $36.4 \%$ of the reviewed literature pointed out this feature as desirable. Odendaal et al (2015), Call et al (2015) and Ly, LaBonte \& Bourgeault (2016) recorded expressions for security and privacy from healthcare providers who were using the mobile phones for healthcare management.

\subsubsection{Intervening and dependent constructs}

Fit: Fit refers to the suitability of an artefact to its intended use (Mburu, Franz \& Springer, 2013). In this study about $63.6 \%$ of the papers reviewed agreed that it is an intervening variable intended to measure the usefulness of a particular mHealth solution in meeting user needs. Mburu, Franz \& Springer (2013) posit that Fit is influenced by potentially negative individual and process requirements. However, these requirements if well addressed using an IT solution; it is possible to significantly improve Fit (Blanas et al, 2014, Call et al (2015) and Odendaal et al, 2015).

Quality of healthcare support: Quality of healthcare support is a measure of overall quality of support provided by a mHealth solution. Parameters used to evaluate healthcare IT innovations include quality (Call et al, 2015), costs (Engle et al, 2015 and Prinja et al 2018)), safety, efficiency and outcomes. However, because quality is more general, we additionally use healthcare quality indicators as proposed by Arah (2006) namely effectiveness, safety, and responsiveness.

\section{Limitations:}

There are several limitations of this review. Firstly, the number of studies was small with generally limited sample sizes. Conclusions about the acceptable mobile app features are therefore tentative. Secondly, the qualitative feedback is based on a small number of healthcare provider studies and generalizing their views to a wider population should be exercised with caution. This feedback is nevertheless informative and highlights the importance of involving healthcare providers in app design. Thirdly, we aimed to reduce publication bias, and although our inclusion criteria were broad, our search was limited to English-language papers. Fourthly, all the articles included in this review originated from work in North America, Africa, and Asia; therefore, these results are limited to the experiences of those regions. However, that geo-spatial mix enriched the analysis, as perceptions on which mobile app features are desirable did not show significant regional variability.

\section{Conclusions:}

The study exposed the healthcare provider's perceptions to the mobile technology attributes as customised requirements, process requirements, moderating information technology constructs and intervening \& dependent requirements. Geospatial setting and income status of the country are less important in shaping the perceptions of healthcare providers as users of the mobile technology in improving healthcare outcomes.

The study has shown that the perception of Healthcare providers on mHealth is good and they can use it to improve health outcomes during emergencies and pandemics. mHealth holds great promise for widening access to health treatment during emergencies and pandemics in low to upper-middle income countries where the challenges of meeting public and personal health needs are considerable. This potential will not be realized unless more future research is conducted in these contexts, hence need to for me to develop, implement and evaluate an application which is compatible in adding new information in emergencies and pandemics. The research will help the HcPs to offer quality HIV management to adolescents and young adults living HIV to improve their health outcomes during emergencies and pandemics like COVID-19.

\section{Annex \\ Outcome after the literature review}

As it was in emergency the researcher developed COVID-19 application for healthcare providers which have been in use since April 2020. The overall effects of the communication application are now under review. 


\section{Developed mhealth application screenshots}
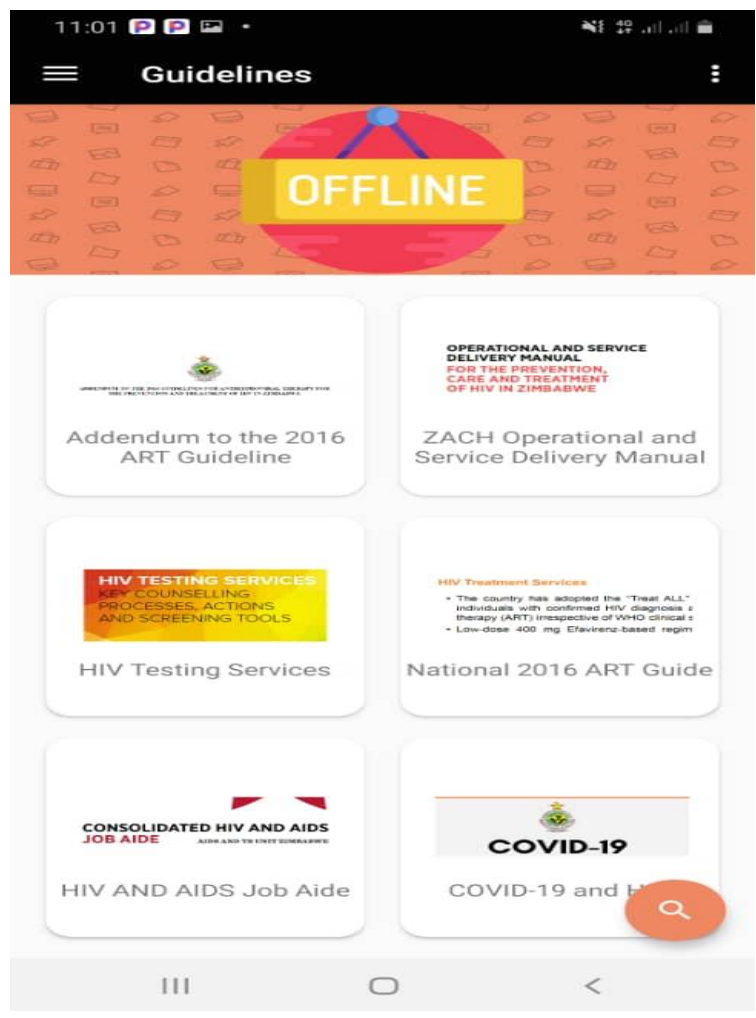

Researcher developed COVID 19 mobile app screenshot 1 , (2021)

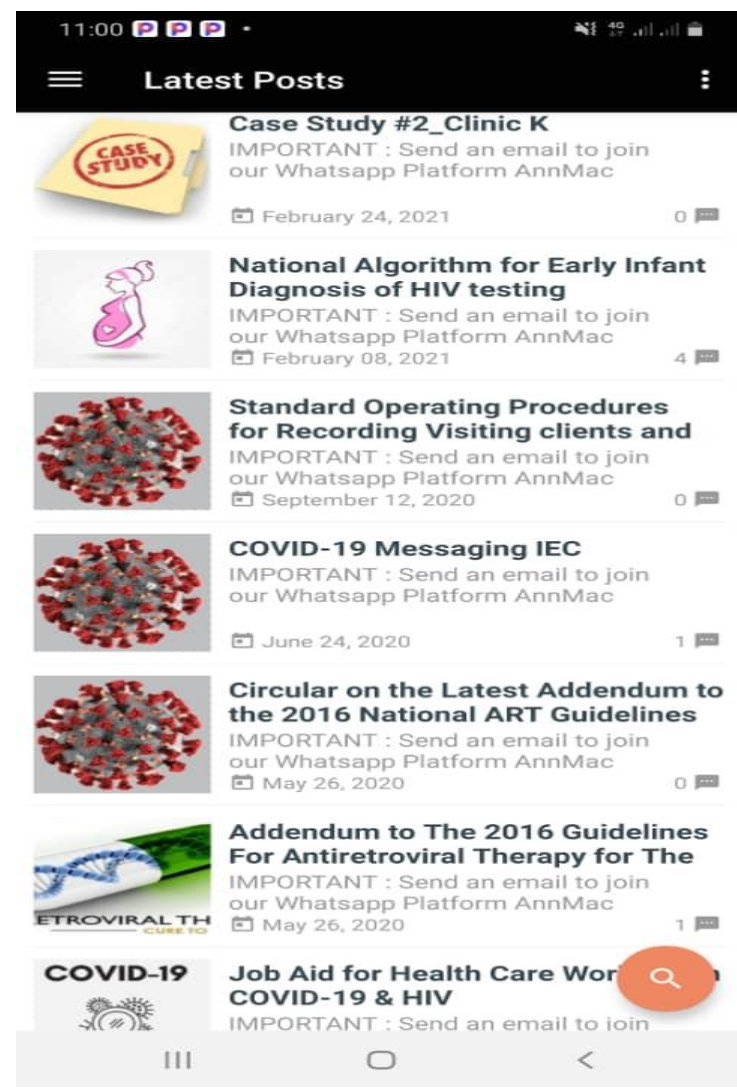

Researcher developed COVID mobile app screenshot 2,

\section{Acknowledgements:}

This research was conducted through desk review as part of the requirements for $\mathrm{PhD}$ offered by the Chinhoyi University of Technology (CUT), Zimbabwe. This came after the training on the Structured Operational Research and Training Initiative (SORT IT), a global partnership led by the Special Programme for Research and Training in Tropical Diseases at the World Health Organization (WHO/TDR). The training model was based on a course developed jointly by the International Union against Tuberculosis and Lung Disease (The Union) and Médecins Sans Frontières (MSF). The specific SORT IT programme resulted in the author enrolling for $\mathrm{PhD}$ through research. The author would want to acknowledge her former employers (MSF-Spain and MSF-Holland), her current employer (US PEPFAR-CDC/ZACH) and the Zimbabwe Ministry of Health and Child Care for all the exposure and support in HIV management. The University had no role in study design, data collection and analysis, decision to publish or preparation of the manuscript. Conflicts of interest: none declared.

\section{References:}

[1] Arah, O. A., Westert, G.P., Hurst, J. and Klazinga. N. S. (2006). A conceptual framework for the oecd health care quality indicators project. International Journal for Quality in Health Care, 18:5 - 13.

[2] Atkins S, Lewin S, Smith $\mathrm{H}$, Engel $\mathrm{M}$, Fretheim A, Volmink J. (2008); conducting a meta-ethnography of qualitative literature: lessons learnt. BMC medical research methodology. 8(1):21. [PubMed: 18416812]

[3] Carroll C, Booth A, Lloyd-Jones M. (2012). Should we exclude inadequately reported studies from qualitative systematic reviews? An evaluation of sensitivity analyses in two case study reviews. Qualitative Health Research. 22(10):1425-1434. [PubMed: 22865107]

[4] Carroll C, Booth A, Leaviss J, Rick J. (2013) "Best fit" framework synthesis: refining the method. BMC Medical Research Methodology.; 
[5] Goodhue, D. L. and Thompson, R. L. (1995). Task-technology fit and individual performance. MIS Quarterly, 19:213 - 236.

[6] International Telecommunication Union (2013). The world in 2013, ICT facts and figures. ICT Data and Statistics Division, Telecommunication Development Bureau Geneva.

[7] Omachonu V. K. and. Einspruch. N. G (2010) Innovation in healthcare delivery systems: A conceptual framework. The Innovation Journal: The Public Sector Innovation Journal, 15(1):1 - 20.

[8] Overby. E. (2008). Process virtualization theory and the impact of information technology. Organization Science, 19(2):277 - 291

[9] Svoronos T, Mjungu D, Dhadialla P, Luk R, Zue C (2010) CommCare: Automated Quality Improvement To Strengthen Community-Based Health The Need for Quality Improvement for CHWs. Available: http://d-tree.org/ wp.../Svoronos-MedinfoCommCare-safe-pregnancy1.pdf. Accessed November 12, 2018.

[10] Tomlinson M, Rotheram-Borus MJ, Swartz L, Tsai AC. (2013) scaling up mHealth: where is the evidence? PLoS Med.10 (2):e1001382.

[11] United Nations (2010). The millennium development goals report. United Nations, New York,

[12] Venkatesh V, Speier C, Morris MG. (2002) User acceptance enablers in individual decision making about technology: toward an integrated model. Decis Sci.33(2):297316.

[13] Venkatesh V, Thong J, Xu X. (2012) Consumer acceptance and use of information technology: extending the unified theory of acceptance and use of technology. MIS Q.; 36(1):157-78.

[14] Vital Wave Consulting (2009). MHealth for Development: The Opportunity of Mobile Technology for Healthcare in the Developing World. Washington, D.C. and Berkshire, UK: UN Foundation-Vodafone Foundation Partnership.
[15] World Health Organization (2011). Mhealth: New horizons for health through mobile technologies: second global survey on ehealth, 2011.

[16] World Health Organization. Coronavirus Disease (COVID-19) Weekly Epidemiological Update and Weekly Operational Update 29 December 2020. Available online: https://www.who.int/emergencies/diseases/ novel-coronavirus-2019/situationreports (accessed on 30 March 2021

[17] Yu, P. Wu, M. X. Yu, H.and Xiao, G. Q (2006). The challenges for the adoption of m-health. In Proc. of IEEE Int. Conf. on Service Operations and Logistics and Informatics, pages $181-186$ 